\title{
Brecha entre Investigación y Praxis Educativas en la Enseñanza de Biología
}

\section{Gap between Educational Research and Praxis in Biological Education}

\author{
Tamara Esquivel-Martín * \\ Beatriz Bravo-Torija \\ José Manuel Pérez Martín
}

Universidad Autónoma de Madrid, España

\begin{abstract}
La división celular es la base conceptual para poder entender los procesos de crecimiento o regeneración de tejidos, así como las enfermedades hereditarias, los problemas reproductivos o el cáncer, que tanto interés despiertan en la población actualmente. Una correcta alfabetización científica pasa por un proceso de enseñanza-aprendizaje que considere los propósitos del desarrollo científicotecnológico y las consecuencias de este en la sociedad y el ambiente. Se analizan las investigaciones educativas que abordan la división celular entre 2000-2017. Y, examina la utilidad que los docentes de Educación Secundaria Obligatoria atribuyen a las publicaciones sobre enseñanza de Biología en su praxis diaria. Para ello, se realiza una búsqueda documental en 14 revistas de Didáctica de Ciencias Experimentales (DCE) y se diseña un cuestionario con 9 preguntas, obteniéndose un total de 117 respuestas. Los resultados muestran que los docentes no suelen consultar artículos en revistas de DCE, y que, en caso de necesitarlo, las temáticas más interesantes son aquellas que proponen actividades de aula y recursos de apoyo; que no se corresponden con las publicaciones de mayor impacto. Se evidencia una débil transferencia desde la investigación educativa en DCE a la práctica docente en las aulas de Biología de ESO, y se discuten en el artículo las posibles causas e implicaciones de una problemática tan significativa en Educación.
\end{abstract}

Descriptores: Enseñanza de la biología; Investigación; Publicación educacional; Enseñanza secundaria; Docente.

Cell division is the conceptual basis to understand processes such as tissue growth or regeneration, as well as inherited diseases, reproductive problems or cancer, which are of great interest to the population nowadays. A correct scientific literacy in this sense needs a teaching-learning process that considers the purposes of scientifictechnological development and its consequences in society and the environment. It analyses several publications about cell division between 2000-2017. And it examines how Secondary Education teachers make use of these investigations in their daily practice. To do so, a documentary research was carried out in 14 Didactic of Experimental Sciences journals and a questionnaire with 9 questions was designed, getting 117 answers. The results show that teachers do not usually read scientific journals publications, and that, if needed, the most interesting topics are those that propose classroom activities and educational resources which are not the publications of greater impact. It is shown a weak transfer from educational research in Didactic of Experimental Sciences to teaching practice in Biology classrooms of Secondary Education. Possible causes and implications of such a significant problem in Education are discussed in the article.

Keywords: Biology; Research; Educational publications; Secondary education; Teacher.

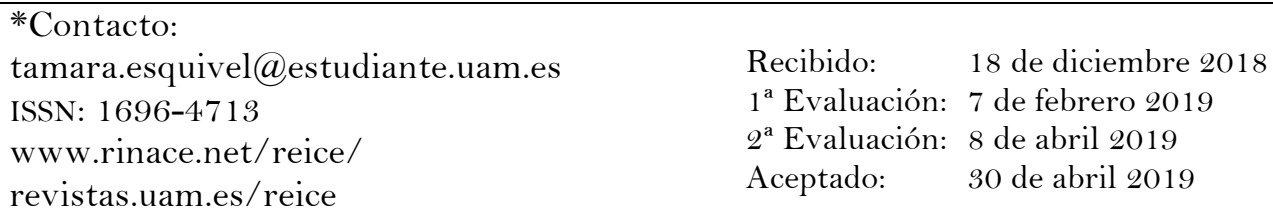




\section{Introducción}

El concepto de investigación educativa es controvertido (Manzano-García, 2016), y muchos docentes desconocen a qué hace referencia exactamente (Murillo y Perines, 2017). Ha sido definida como un proceso durante el cual los investigadores se cuestionan acerca de una problemática o situación en el ámbito educativo, la acotan, analizan y formulan propuestas de cambio (Martínez, 2007), siendo su principal finalidad la mejora de la práctica de la enseñanza (Jiménez-Aleixandre, 2008; Oliva, 2017; Sancho, 2010). Pero esta transformación no puede darse sin transferencia (Muñoz Martínez y Garay, 2015; Wandersman et al., 2008), entendiéndose a esta como la aplicación de los resultados de la investigación educativa en la práctica docente de aula (Lara, Förster y Gorichon, 2018; Lavis et al., 2003). Por tanto, si su calidad y transferencia son adecuadas, la investigación educativa es una herramienta para lograr el cambio educativo en la enseñanza en general (Gallardo et al., 2017), y de las Ciencias en particular (Blanco-López, Martínez-Peña y Jiménez-Liso, 2018; Porlán, 2018), que necesita la sociedad actual.

Sin embargo, los docentes de otras etapas educativas consideran que los investigadores de las universidades se encuentran muy alejados de la realidad de las aulas, lo que supone un impedimento al diseñar propuestas verdaderamente útiles en su práctica diaria y obstaculiza el cambio educativo (Broekkamp y Van Hout-Wolters, 2007; Ion y Iucu, 2014; Muñoz-Repiso, 2004; Williams y Coles, 2007). Por tanto, es necesario conectar los intereses de investigadores y docentes en activo de los diferentes niveles educativos. A fin de salvar la distancia entre la producción del conocimiento y su repercusión práctica surgió el concepto de Movilización del Conocimiento (Knowledge Mobilization) en el contex to internacional (Cooper, Rodway y Read, 2018), haciendo hincapié en la relevancia de mejorar las estrategias de difusión y comunicación para generar un impacto mayor del que se está produciendo. Sin embargo, a la hora de publicar las investigaciones, los criterios de calidad que condicionan el impacto de las revistas dificultan la transferencia del conocimiento a la práctica docente (Oliva, 2017).

\section{Revisión de la literatura}

La brecha existente entre investigación y práctica educativas en general (Gore y Gitlin, 2007; Murillo y Perines, 2017; Sancho, 2010), y en didáctica de Ciencias en particular (Blanco-López, Martínez-Peña y Jiménez-Liso, 2018; Russell y Martin, 2014; Sanmartí, 2008; Solbes y Gavidia, 2013), se menciona en estudios que implican a docentes de diversas partes del mundo, incluido nuestro país. Además, la percepción negativa en torno a la investigación educativa no es novedosa, pues ya Kaestle (1993) hablaba de su pésima reputación, y otros autores como Vanderlinde y van Braak (2010) o Perines (2018) siguen haciendo referencia a la puesta en duda de su utilidad por el profesorado. Esto se debe a que las temáticas estudiadas muchas veces no se ajustan a la demanda docente, y su pertinencia es discutible al no ser consideradas las propuestas aplicables al aula (Broekkamp y Van-Hout-Wolters, 2007; Gore y Gitlin, 2007; Ion y Iucu, 2014; Murillo y Perines, 2017; van Schaik et al., 2018).

Otro condicionante que afecta a la desconexión existente entre investigación y praxis educativas es la promoción laboral, principal motor de actuación del personal experto investigador en las universidades (Murillo y Perines, 2017; Perines, 2018). En este sentido, los investigadores persiguen publicar en revistas de prestigio indexadas en 
Journal Citation Reports (JCR) o Scopus (Scimago Journal Rank, SJR), al ser el impacto de las publicaciones el criterio para evaluar la actividad científica en cualquier disciplina (Galán y Zych, 2011; Ruiz García y Valero Aguayo, 2018). En España es la Agencia Nacional de Evaluación de la Calidad y Acreditación (ANECA) la encargada de dicha evaluación para el acceso a las distintas figuras de profesor universitario, establecidas en la Ley Orgánica de Modificación de la Ley Orgánica de Universidades (LOMLOU) de 2007.

Lo anterior hace que el principal interés de los investigadores universitarios muchas veces entre en conflicto con el de los docentes de ESO, futuros consumidores de su producción científica en pos del cambio educativo. Estos últimos están interesados principalmente en trabajos de innovación educativa, entendida como creación y difusión de herramientas nuevas, prácticas, sistemas de organización o tecnologías (Foray y Raffo, 2012) que revaloricen los procesos ocurridos en la institución educativa y mejoren los resultados de los aprendizajes, la satisfacción de los implicados, o ambas cosas a la vez (OCDE, 2009; Pedró, 2015). Estas demandas, en el caso concreto de las Ciencias, pretenden mejorar las dificultades de su enseñanza-aprendizaje, entre otras razones por la desmotivación del alumnado. Así, según Robles y otros (2015), las metodologías tradicionales empleadas en su formación son una de las causas de su actitud negativa, por lo que parecería sensato que la investigación educativa se centrase en la propuesta de innovaciones. A pesar de ello, autores como Oliva (2005) y de Jong (2007) señalan que un criterio de calidad de las revistas científicas es presentar un mayor porcentaje de trabajos de investigación frente a los trabajos de innovación educativa. Esto es un reflejo de la diferencia de estatus que se le otorga a la innovación educativa, que debiera ser considerada como la investigación educativa aplicada.

En el caso del área de conocimiento de Didáctica de las Ciencias Experimentales, se precisa un cambio educativo que mejore esta situación. Según Murillo y Krichesky (2012), el cambio educativo implica la mejora constante del proceso de enseñanza-aprendizaje y de sus resultados. Para ello, es preciso producir cambios en la organización escolar, impulsar innovaciones didácticas, y atender a los intereses de los docentes, vinculando las investigaciones a las necesidades del aula. Esto requiere la implicación activa del profesorado como consumidor y productor activo de dicha investigación (Sanmartí, 2008). Sin embargo, mientras que en disciplinas como Física y Química (Solbes et al., 2013) o Química (Wissiak y Ferk, 2013), existen estudios que se cuestionan si los docentes incorporan los resultados de las investigaciones en didáctica, existen pocas investigaciones que estudien la praxis del profesorado en la enseñanza de las Ciencias naturales (Gutiérrez, Muñoz de Corrales y del Campo, 2017). Así, hay contenidos de Biología que requieren de un cambio educativo que por el momento no ha sido analizado en profundidad, como es el caso de la enseñanza de la división celular. Esta necesidad es evidente si consideramos los estudios de ideas alternativas de estudiantes de ESO sobre este contenido que se han venido realizando de manera histórica (Lewis, Leach y WoodRobinson, 2000; Ruíz-González, Banet y López-Banet, 2017). Sus resultados muestran que a lo largo del tiempo han persistido creencias como la falta de cromosomas en otras especies; la confusión entre los procesos mitótico y meiótico o entre las estructuras celulares animales y vegetales implicadas; así como el desconocimiento de la carga cromosómica en cada fase del ciclo celular. Esto sugiere que las propuestas realizadas desde la investigación educativa para mejorar la situación no la están resolviendo. Una posible causa es que los profesores no hagan uso de ellas ya que, según Oliva (2011) su actuación docente predominante se basa en un modelo artesanal-individualista, 
considerando únicamente su experiencia personal sin tomar como referencia las publicaciones relacionadas con la temática. Esto nos lleva a preguntarnos: ¿por qué los artículos de investigación educativa en Biología no están siendo considerados como recursos en la preparación de sus clases?

Solbes y colaboradores (2018) han puesto de manifiesto que los docentes de secundaria de Ciencias no consideran útiles los artículos de investigación educativa, entre otras razones, porque sus temáticas están desconectadas de la realidad del aula y no se proponen actuaciones aplicables en su día a día. Si bien es cierto, existen propuestas de aula que tratan de conectar la investigación y la praxis educativa en Biología, como aquellas que proponen el uso del enfoque Científico, Tecnológico, Social y Ambiental (CTSA). Este aboga por el desarrollo personal y social para un futuro sostenible, y reconoce la necesidad de hacer consciente a la población de los problemas existentes en una sociedad llena de desequilibrios políticos, económicos, culturales y ambientales como la nuestra (Pérez Martín y Bravo-Torija, 2018; Strieder, Bravo-Torija y Gil-Quílez, 2017). En este sentido, la enseñanza de las divisiones celulares es especialmente relevante, ya que es el eje vertebrador para la comprensión de fenómenos como la reproducción de los seres vivos, el crecimiento de los organismos pluricelulares, la regeneración de tejidos o la herencia genética. Asimismo, permite entender los problemas reproductivos, el cáncer o su posible relación con la presencia de contaminantes tóxicos en el entorno. Lo que evidencia que tiene implicaciones en temas controvertidos de corte ambiental y sanitario. Por ello, su correcto aprendizaje es clave en la alfabetización científica crítica, esencial para tomar decisiones responsables en cuestiones que generan controversia, y participar libremente en una sociedad democrática (Gil y Vilches, 2017; Harlen, 2015). Porlán (2018) lo denomina democracia epistemológica, lo que estaría estrechamente ligado con el concepto de Justicia Social (Murillo y Hernández-Castilla, 2011).

Un ejemplo concreto del uso del enfoque CTSA en la enseñanza de las divisiones celulares, se muestra en el estudio de Domènech-Casal (2016), en el que se plantea una actividad basada en la indagación sobre la mitosis y el cáncer. En ella los estudiantes vivencian el proceso investigador, pudiendo complementarse estas dinámicas de construcción de conocimiento con reflexiones acerca de la importancia de financiar la investigación contra el cáncer. De igual forma, en el estudio de Pérez Martín y Bravo-Torija (2018), se presentan intervenciones de aula en diferentes niveles educativos donde se relacionan los tóxicos ambientales con alteraciones celulares que causan enfermedades, haciendo partícipe al alumnado en la búsqueda de soluciones para minimizar la problemática ambiental en su vida cotidiana.

En este sentido, el presente estudio, que forma parte de una investigación más amplia encaminada a la mejora de la enseñanza de la división celular, busca dar respuesta a la siguiente pregunta: ¿En qué medida la investigación educativa en la enseñanza de Biología realizada en la universidad llega realmente a las aulas y al profesorado en ejercicio de Educación Secundaria Obligatoria? De esta derivan otras dos preguntas investigables más específicas: ¿Qué implicaciones tiene el impacto de las revistas en la investigación educativa universitaria? ¿Qué necesidades manifiestan los docentes para el cambio educativo cuando son preguntados por su praxis?

Las tres preguntas de investigación anteriores se concretan en los siguientes objetivos: a) identificar cuáles son los enfoques predominantes en los artículos sobre la enseñanzaaprendizaje de las divisiones celulares de las revistas de mayor impacto (investigación 
educativa); b) examinar qué recursos y temas de investigación presentan mayor interés para los docentes de Biología de ESO (praxis educativa); y c) reflexionar acerca de cómo se articulan investigación y praxis educativa en la enseñanza de la Biología a la luz de los resultados obtenidos.

\section{Método}

La recogida y el análisis de los datos se centró en dos aspectos fundamentales: a) el estado de la investigación educativa en relación con la división celular desde principios de siglo, para lo cual se analizaron los hallazgos en la literatura sobre la enseñanza-aprendizaje de este contenido entre 2000-2017; y b) la opinión de los docentes de Biología de ESO en activo acerca del producto de las investigaciones sobre la enseñanza de Biología, y grado en que las usan, para lo cual se implementó un cuestionario durante el curso 2018/2019.

2.1. Revisión de la literatura sobre el proceso de enseñanza-aprendizaje de la división celular en revistas de Didáctica de Ciencias Experimentales (DCE) en el período 2000-2017

La búsqueda documental se realizó en 14 revistas relevantes en DCE a nivel nacional e internacional, 10 de ellas con impacto en JCR: Enseñanza de las Ciencias, International Journal of Science and Mathematics Education, International Journal of Science Education, Journal of Biological Education, Journal of Research in Science Teaching, Journal of Science Education and Technology, Research in Science Education, Science Education, Scientific Research and Essay, y The American Biology Teacher; y cuatro de ellas sin impacto en JCR: Alambique, Didáctica de las Ciencias Experimentales, Didáctica de las Ciencias Experimentales y Sociales, Revista Electrónica de la Enseñanza de las Ciencias, y Revista Eureka Sobre Enseñanza y Divulgación de las Ciencias, aunque esta última está indexada en el segundo cuartil de SJR. Las palabras clave empleadas fueron: "mitosis", "meiosis" y "cell division/división celular”. Se recopilaron 63 artículos que pueden consultarse en este enlace: http://bit.ly/2I6HNMB.

Tras su selección se analizaron:

- La mención expresa de los términos división celular, mitosis o meiosis en el título, en español o inglés; ya que el título es la primera etiqueta que atrae al lector (Murillo, Martínez-Garrido y Belavi, 2017)

- El nivel educativo al que se destinan los estudios, ya sean ESO y/o Bachillerato, o etapas superiores (grado y posgrado);

- La temática abordada y su evolución en el tiempo. Las temáticas de los artículos fueron categorizadas en función del objetivo de investigación al que respondían los estudios: i) detección de ideas alternativas, ii) propuesta de actividades y recursos, iii) propuesta de aplicación de modelización, iv) análisis del conocimiento didáctico del contenido (CDC), y v) presentación de progresiones de aprendizaje;

- El factor de impacto JCR de los artículos en el año en que fueron publicados.

Además, a fin de evaluar la relación entre dichas variables, se realizaron análisis descriptivos e inferenciales (correlaciones, $\chi^{2}$ ) con Microsoft ExcelTM (2016, EEUU) e IBM ${ }$ SPSS $®$ Statistics 23 (2015, EEUU). 


\subsection{Cuestionario}

Los participantes voluntarios del estudio fueron 117 docentes españoles autoseleccionados a través de la difusión de un cuestionario en Redes Sociales, y codificados desde P1 hasta P117. Para el muestreo no probabilístico o dirigido se fijaron tres criterios: a) el nivel educativo: ESO; b) la condición laboral de estar en activo, y c) la especialización del profesorado en Biología. La vía de participación se basaba en dar respuesta a un formulario de 9 preguntas, algunas abiertas y otras de escala $0-10$, disponible online: https://goo.gl/y7Y5JP. Este cuestionario, antes de aplicarse en la muestra piloto, fue sometido al juicio de tres expertos del área de conocimiento (Didáctica de las Ciencias Experimentales) para evaluar la claridad, pertinencia y extensión de los ítems. Estos se centraban fundamentalmente en: a) conocer el material de preferencia del profesorado para la preparación de sus clases, el idioma en que prefieren consultarlo, y la utilidad que le otorgan al recurso; b) conocer la percepción del profesorado acerca de la utilidad de las publicaciones sobre las temáticas anteriormente mencionadas; y c) determinar su grado de conocimiento acerca de las revistas científicas a las que tienen acceso desde el centro educativo donde desempeñan su labor.

\section{Resultados}

\subsection{Resultados del análisis documental de artículos relacionados con la división celular}

El análisis descriptivo de los 63 artículos relacionados con la división celular se llevó a cabo atendiendo a los descriptores antes mencionados (título, nivel educativo, evolución de las temáticas e impacto de las publicaciones), y reveló, en primer lugar, que solo el $30 \%$ de las publicaciones contenían los términos división celular (cell division), mitosis o meiosis en el título. Además, la mayoría de los estudios (61,9\%) se centran en la etapa de ESO y Bachillerato.

Respecto a la evolución de las temáticas en el tiempo (figura 1), podemos ver cómo, dentro de los artículos de la muestra estudiada, a principios de siglo predominaban las investigaciones acerca de las concepciones del alumnado sobre contenidos relacionados con la división celular; pero con el paso del tiempo este tipo de estudios han ido disminuyendo. Por su parte, los estudios que presentaban y proponían progresiones de aprendizaje surgieron más recientemente, siendo el primero de nuestra muestra de estudio el de Duncan, Rogat y Yarden (2009). Con respecto a las propuestas de aplicación de la modelización, al igual que los estudios de ideas alternativas, fueron frecuentes a principios de siglo como la de Clark y Mathis (2000), y más recientemente como la de Eliyahu (2014). Sin embargo, los estudios como el de González y Rossi (2015) que analizaban el CDC predominaron en el trienio 2009-11, siendo actualmente la segunda temática con más publicaciones. Finalmente, los artículos donde se proponen actividades y recursos digitales como los de Esteve, Baños-González y Moreno (2017), o Íñiguez-Porras y Puigcerver-Oliván (2013), han sido constantes en el periodo estudiado. 


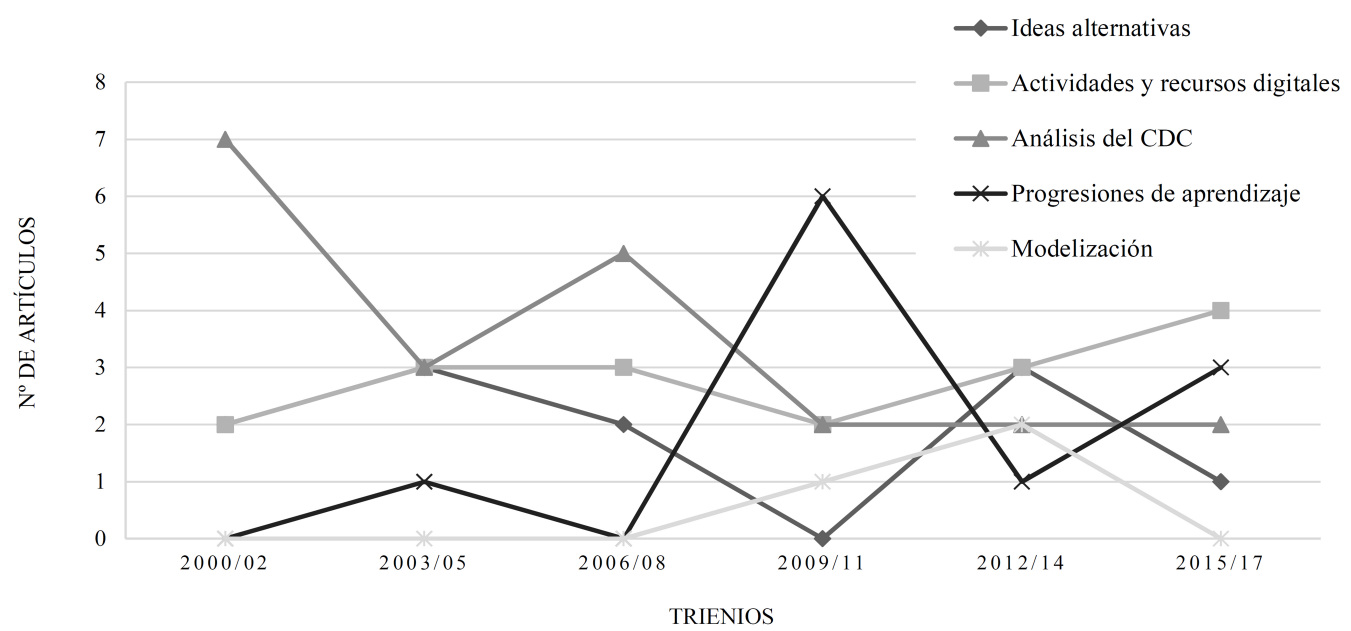

Figura 1. Evolución de las temáticas o enfoques abordados en los artículos por trienios Fuente: Elaboración propia.

Por otro lado, el 68,3\% de publicaciones presentan impacto bajo (Q4) o nulo, teniendo solo el 22,2 \% un impacto elevado en JCR ( 1 y Q2). Además, si enfrentamos los años de publicación al impacto de los artículos en las diferentes revistas, encontramos que en el último trienio hay más publicaciones en relación con la división celular en revistas de DCE sin indexar en JCR (cuadro 1).

Cuadro 1. Frecuencias absolutas de las publicaciones por trienios desde 2000, distribuidas por impacto.

\begin{tabular}{|c|c|c|c|c|c|c|c|}
\hline \multirow{2}{*}{ Cuartiles } & \multicolumn{6}{|c|}{ TrIEnIOS } & \multirow{2}{*}{ TOTAL } \\
\hline & $2000 / 02$ & $2003 / 05$ & $2006 / 08$ & $2009 / 11$ & $2012 / 14$ & $2015 / 17$ & \\
\hline Q1 JCR & 1 & 0 & 0 & 1 & 2 & 1 & 5 \\
\hline $\mathrm{Q}^{2} \mathrm{JCR}$ & 2 & 3 & 0 & 2 & 0 & 2 & 9 \\
\hline Q3 JCR & o & 1 & 1 & 2 & 2 & o & 6 \\
\hline $\mathrm{Q}^{4 \mathrm{JCR}}$ & 7 & 3 & 6 & 4 & 5 & 1 & 26 \\
\hline $\begin{array}{l}\text { Sin indexar } \\
\text { en JCR }\end{array}$ & 1 & 3 & 3 & 2 & 2 & 6 & 17 \\
\hline Total & 11 & 10 & 10 & 11 & 11 & 10 & 63 \\
\hline
\end{tabular}

Fuente: Elaboración propia.

Por su parte, el análisis estadístico inferencial de los datos mostró una correlación significativa ( $\mathrm{r}$ de Pearson $=-0,376, \mathrm{p} \leq 0,05)$ entre el cuartil $(\mathrm{Q})$ en JCR de las revistas en el año en que se publicaron los artículos, y las temáticas de estos. Lo anterior implica que la distribución de las temáticas no es homogénea entre los diferentes cuartiles de impacto $\left(\chi^{2}, \mathrm{p} \leq 0,05\right)$. Concretamente, el $60 \%$ de las publicaciones en las revistas de mayor impacto (Q1) tratan las progresiones de aprendizaje y el análisis del CDC, sin que aparezcan publicaciones sobre modelización. Sin embargo, tanto esta temática como la propuesta de actividades predominan en las revistas de $\mathrm{Q}_{4} \mathrm{y}$ sin impacto en JCR (cuadro 2). 
Cuadro 2. Porcentaje de temáticas asociado a los distintos cuartiles de impacto de las revistas consultadas

\begin{tabular}{lcrrrr}
\hline & Q1 & $\mathbf{Q 2}^{2}$ & $\mathbf{Q 3}^{3}$ & $\mathbf{Q 4}^{4}$ & SIN INDEXAR \\
\hline Modelización & 0 & 0 & 16,7 & 30,8 & 11,8 \\
Actividades y recursos digitales & 20 & 33,3 & 0 & 23,1 & 41,2 \\
Ideas Alternativas & 20 & 33,3 & 50 & 30,8 & 35,3 \\
Análisis del CDC & 20 & 33,3 & 16,7 & 15,4 & 11,8 \\
Progresión de aprendizaje & 40 & 0 & 16,7 & 0 & 0 \\
\hline Total & 100 & 100 & 100 & 100 & 100 \\
\hline
\end{tabular}

Fuente: Elaboración propia.

\subsection{Resultados de del cuestionario realizado a docentes de Biología en ESO}

Fuentes de consulta preferidas por los docentes, idioma y utilidad otorgada al recurso

Las dos principales fuentes de consulta de los participantes son Internet $(51,3 \%)$ y los libros de texto $(41,9 \%)$. Solo el $3,4 \%$ de los docentes afirma emplear revistas científicas y/o didácticas durante la preparación de sus clases. En cualquier caso, al 77,8\% del profesorado les interesan los materiales que están en castellano, seguido de un $15,4 \%$ que los prefieren en inglés. Cuando puntuaron la utilidad que le otorgaban al recurso de primera elección (recursos de internet, libros de texto, revistas científicas y/o didácticas u otros) que habían seleccionado en el cuestionario, se obtuvo como valor moda 7 y como valor medio 7,9 con una desviación estándar de 1,2.

Grado de utilidad para el profesorado de cinco temáticas distintas publicadas en revistas de investigación en Didáctica de Ciencias Experimentales

$\mathrm{Si}$ analizamos las preferencias docentes al recurrir a revistas de investigación en DCE, nos encontramos con que las diferentes temáticas no son consideradas igual de valiosas por los profesores de ESO participantes (ANOVA, post hoc Tukey p $\leq 0,05$ ). Así, estos (cuadro 3) consideran que los artículos que tratan sobre progresiones de aprendizaje y conocimiento didáctico del contenido son significativamente menos útiles que los estudios de ideas alternativas, las propuestas de modelización o las de actividades y recursos, siendo estas tres últimas las temáticas mejor valoradas. Tanto es así que tanto el valor moda como el valor medio de utilidad otorgado a las propuestas de actividades y recursos digitales (cuadro 3) supera al del material de primera elección, pues el 46,2\% del profesorado ha puntuado esta temática mejor que al recurso que suele emplear.

Cuadro 3. Valoración de la utilidad de las temáticas por el profesorado: medidas de centralidad y dispersión

\begin{tabular}{lrc}
\hline & MODA & MEDIA \pm DS \\
\hline Propuestas de modelización & 9,0 & $7,9 \pm 1,2$ \\
Propuestas de actividades y recursos digitales & 9,0 & $7,2 \pm 2,3$ \\
Estudios de ideas alternativas & 8,0 & $8,0 \pm 2,0$ \\
Estudios sobre el CDC & 7,0 & $7,3 \pm 2,1$ \\
Progresiones de aprendizaje & 6,0 & $6,9 \pm 2,4$ \\
\hline
\end{tabular}

Fuente: Elaboración propia. 


\subsection{Grado de conocimiento acerca de las revistas científicas a las que tienen acceso desde el centro educativo donde desempeñan su labor}

Los resultados muestran que el 53\% de los docentes desconocen si los centros educativos en los que trabajan están suscritos a alguna revista científica (P81: "no lo he preguntado nunca, no lo sé”), dejando entrever desinterés por saberlo, y un 17,1\% afirman que no están suscritos a ninguna (P39: "no estamos suscritos a revistas, ni es una opción que estemos contemplando”). Alambique o Enseñanza de las Ciencias son algunas de las respuestas más frecuentes del resto de profesores. Además, entre los resultados se ven incluidos buscadores de publicaciones científicas o de lecciones multimedia de Ciencias para ESO.

\section{Discusión y conclusiones}

El objetivo final de esta investigación era reflexionar acerca de cómo se articulan investigación y praxis educativas en la enseñanza de la Biología, tras conocer cuáles son los enfoques predominantes de los artículos que abordan la enseñanza-aprendizaje de las divisiones celulares en las revistas de más impacto, así como qué recursos y temas de investigación presentan mayor utilidad para los docentes de Biología de ESO. De este modo, tras profundizar en la brecha existente entre teoría y praxis en la educación en Ciencias (Sanmartí, 2008), y, por ende, en el percibido divorcio entre investigadores y profesores de ESO (Oliva, 2012), se hace necesario discutir las posibles razones por las cuales la investigación educativa universitaria en relación con la división celular no está logrando promover el cambio educativo que necesitan nuestros estudiantes. Esta situación es patente en aquellos estudios que muestran cómo el alumnado continúa manifestando concepciones alternativas en relación con contenidos básicos de Biología que obstaculizan su sentido crítico al respecto, a pesar de la existencia de investigaciones que han abordado dicha problemática en los últimos años. Lo que nos lleva a plantearnos: ¿por qué los docentes no las están consultando y/o aplicando?

En primer lugar, si nos centramos en el título de las publicaciones, al ser un posible condicionante de su lectura (Murillo, Martínez-Garrido y Belavi, 2017), debería ser lo suficientemente atractivo, estar relacionado con la temática tratada y conectado con la realidad del aula. En este sentido, destacamos que, a pesar de que en todos los artículos analizados se abordan contenidos relacionados con la división celular, solo el 30\% de sus títulos presentan los términos: mitosis, meiosis o división celular, lo que podría contribuir a que el profesorado interesado pase por alto el $70 \%$ restante.

Por otro lado, el hecho de que la mayoría de las publicaciones relacionadas con la división celular se centren en los niveles educativos de ESO y Bachillerato tiene sentido si se analizan los currículos de ambas etapas donde, excepto en $2^{\circ}$ de ESO, se abordan estos contenidos (Real Decreto 1105/2014). No obstante, es igualmente oportuna la revisión que se hace del estado de la cuestión en otros niveles, al haberse detectado dificultades de aprendizaje incluso en estudiantes universitarios y profesorado de Educación Primaria y Secundaria en ejercicio (Pérez Martín y Aquilino, 2017). A fin de solventar dichas dificultades para lograr el aprendizaje integral de los contenidos relacionados con los procesos mitótico y meiótico, el análisis de la evolución de las temáticas en el tiempo pone de manifiesto que se han publicado numerosos trabajos de investigación e innovación educativa encaminados a la mejora de la práctica docente en la enseñanza de la división 
celular. En los dos últimos trienios predominan aquellos donde se realizan propuestas de actividades y recursos digitales, si bien es cierto, en revistas de bajo o nulo impacto en JCR.

Sin embargo, los docentes no están incorporando los resultados de la investigación didáctica en las aulas de Biología (Greca y El-Hani, 2015). Para cerrar esta brecha es necesario empezar por conocer la opinión del profesorado acerca de las recomendaciones realizadas desde la investigación en didáctica de las Ciencias. Los resultados de nuestro cuestionario evidencian una notable preferencia por materiales en castellano $(77,8 \%)$, si bien es cierto la literatura mejor considerada está escrita en inglés (Murillo, MartínezGarrido y Belavi, 2017). Además, aunque los docentes consideran que las temáticas abordadas en las revistas científicas y/o didácticas son útiles, a la hora de preparar sus clases prefieren consultar mayoritariamente otras fuentes de internet o libros de texto, por lo que muchos podrían permanecer desactualizados y desorientados en cuanto a las innovaciones técnico-pedagógicas que van surgiendo (Lupión Cobos y Martín Gámez, 2016; Oliva, 2011; van Schaik et al., 2018).

Por ello, se hace imprescindible trasladar a los docentes los productos de las investigaciones educativas que tratan de mejorar la enseñanza-aprendizaje de contenidos difícilmente abordables en el aula como la división celular. En este sentido, existen estudios sobre progresiones de aprendizaje o sobre el conocimiento didáctico del contenido por el profesorado de Ciencias (Magnusson, Krajcik y Borko, 1999; Talanquer, 2013), que se ven enmarcados en un concepto de investigación más teórica. A la luz de los resultados obtenidos en nuestro estudio, estas son las temáticas más frecuentes en revistas indexadas en JCR en el primer o segundo cuartil ( $Q 10$ Q2). Sin embargo, esto se aleja de la realidad de las aulas y del verdadero interés de los docentes en la línea de lo señalado por Bartels (2003), Morales (2010) o Murillo y Perines (2017), quienes aseguran que el traslado de la innovación educativa a la práctica es aún más difícil cuando se trata de reflexiones teóricas y constructos hipotéticos. Así, el profesorado que ha realizado nuestro cuestionario demanda fundamentalmente propuestas de actividades, recursos digitales y modelos que les sirvan como guía al enseñar Biología, seguido de estudios sobre detección de ideas alternativas, ya que todos ellos les podrían resultar de interés por su posible aplicación en el aula. Sin embargo, estas publicaciones centradas en la innovación educativa en relación con la división celular aparecen con mayor frecuencia en las revistas de bajo o nulo impacto en JCR. Estos hallazgos van en la línea de lo descrito por Oliva (2017), quien señala que los estándares de calidad que demandan las bases de datos más exigentes llevan a los investigadores a priorizar el sentido académico frente al profesional ante la evidente competitividad existente en el ámbito educativo, orientando las revistas de DCE fundamentalmente hacia la investigación en lugar de hacia la innovación. Por ende, las publicaciones de investigación educativa aplicada no tienen a priori el prestigio que se le requiere a los docentes universitarios para su promoción laboral. Esto podría entenderse como falta de democracia en la toma de decisiones a la hora de investigar; lo que se opone a la esencia de una sociedad que defiende la Educación para la Justicia Social (Belavi y Murillo, 2016). Se precisa, por tanto, un equilibrio que concilie ambas prácticas, la promoción profesional y la atención al interés del profesorado durante el ejercicio de la investigación educativa.

Por otra parte, a pesar de que muchos docentes reconocen que gracias a internet las posibilidades de acceso a toda la información precisada se han disparado, se quejan de las dificultades para consultar publicaciones de pago (Murillo y Perines, 2017). Lo anterior pone de manifiesto un desconocimiento generalizado acerca de buscadores, bases de datos, 
revistas específicas de didáctica (Solbes et al., 2018; Solís, 2011) y otros recursos disponibles para la consulta de un volumen muy extenso de producción científica en abierto. Esto se traduce en una necesidad de mejora de la formación del profesorado a la hora de investigar y buscar información rigurosa durante sus estudios de grado y posgrado (Pérez Martín y Martínez Luna, 2017). Además, nuestros resultados dejan entrever un desinterés de los docentes por conocer las posibilidades de acceso a revistas de pago en los centros educativos donde ejercen su labor.

Por tanto, el presente estudio ofrece una perspectiva nueva sobre la Justicia Social en la enseñanza de las Ciencias en un contexto inexplorado hasta ahora: las divisiones celulares. No obstante, considerando el alcance de la muestra, sería interesante ampliar el número de participantes en posteriores investigaciones incorporando docentes de otros países, para poder analizar la universalidad del fenómeno por influencia de la variable sociodemográfica. Asimismo, dadas las características del instrumento de recogida de datos, nuestro cuestionario podría complementarse con otro tipo de herramientas de investigación como entrevistas personales. Con ello, se estarían iniciando nuevos trabajos que nos permitirían profundizar en las preferencias docentes en torno a los contenidos más difíciles de abordar en las aulas de Biología hoy en día. Estos resultados podrían compararse con los de nuevas revisiones bibliográficas sobre lo investigado en DCE, en la línea de lo realizado en el presente estudio.

Por último, considerando que debería tenderse a una difusión del conocimiento en abierto, las revistas de impacto deberían publicar más propuestas que respondan a las principales demandas de los docentes en activo, lo que sería una forma de contribuir al cambio educativo, ya que las convierte en verdaderos espacios de socialización para compartir prácticas exitosas de interés (Gallardo et al., 2017).

Por todo lo expuesto, es fundamental que exista un diálogo permanente bidireccional entre docentes universitarios (investigadores) y docentes de otros niveles educativos para compartir sus conocimientos, así como para reflexionar acerca de la práctica profesional de todos los implicados y poder mejorarla. Al estar los centros de interés y difusión de la investigación didáctica universitaria completamente condicionados en la sociedad actual por la "ley del prestigio" en nombre de la calidad educativa, consideramos absolutamente necesaria una reevaluación de los objetivos de la investigación educativa. Por un lado, esta debe dejar de ser una colección constante de papeles que certifiquen méritos, pasando a ser requisito imprescindible que todos sus resultados sean transferibles a la realidad de las aulas; y por otro, los criterios de calidad de las revistas deben promover la equiparación del estatus entre investigación e innovación educativas.

\section{Agradecimientos}

Esta investigación y la difusión de sus resultados está financiada por los proyectos de $\mathrm{I}+\mathrm{D}+\mathrm{i}$ con códigos EDU2017-82688-P y EDU2015-66643-C2-2-P, subvencionados por el Ministerio de Economía y Competitividad. 


\section{Referencias}

Bartels, N. (2003). How teachers and researchers read academic articles. Teaching and Teacher Education, 19(7), 737-753. https://doi.org/10.1016/j.tate.2003.06.001

Belavi, G. y Murillo, F. J. (2016). Educación, democracia y justicia social. Revista Internacional de Educación para la Justicia Social, 5(1), 13-34. https://doi.org/10.15336/riejs2016.5.1

Blanco-López, A., Martínez-Peña, B. y Jiménez-Liso, M. R. (2018). ¿Puede la investigación iluminar el cambio educativo? Ápice. Revista de Educación Científica, 2(2), 15-28. https://doi.org/10.17979/arec.2018.2.2.4612

Broekkamp, H. y Van Hout-Wolters, B. (2007). The gap between educational research and practice: A literature review, symposium, and questionnaire. Educational Research and Evaluation, 13(3), 203-220. https://doi.org/10.1080/13803610701626127

Clark, D. C. y Mathis, P. M. (2000). Modeling mitosis y meiosis: A problem-solving activity. The American Biology Teacher, 62(3), 204-206. https://doi.org/10.2307/4450874

Cooper, A., Rodway, J. y Read, R. (2018). Knowledge mobilization practices of educational researchers across Canada. Canadian Journal of Higher Education, 48(1), 1-21. https://doi.org/10.7202/1050839ar

Domènech-Casal, J. (2016). Drug research: Una secuencia contextualizada de indagación sobre mitosis, cáncer y creación del conocimiento científico. Investigación en la Escuela, 88, 93-111. https://doi.org/10.12795/IE.2018.i88.06

Duncan, R. G., Rogat, A. D. y Yarden, A. (2009). A learning progression for deepening students' understandings of modern genetics across the $5^{\text {th }}-10^{\text {th }}$ grades. Journal of Research in Science Teaching, 46(6), 655-674. https://doi.org/10.1002/tea.20312

Eliyahu, D. (2014). Chromoseratops meiosus: A simple, two-phase exercise to represent the connection between meiosis y increased genetic diversity. The American Biology Teacher, 76(1), 53-56. https://doi.org/10.1525/abt.2014.76.1.11

Esteve, P., Baños-González, I. y Moreno P. P. (2017). ¿Qué pasa a nivel microscópico? Alambique: Didáctica de las Ciencias Experimentales, 88, 27-33.

Foray, D. y Raffo, J. (2012). Business-driven innovation: Is it making a difference in education? An analysis of educational patents. París: OCDE Publishing. https://doi.org/10.1787/19939019

Galán, A. y Zych, I. (2011). Análisis de los criterios de la comisión nacional evaluadora de la actividad investigadora (CNEAI) para la concesión de los tramos de investigación en Educación. Bordón. Revista de Pedagogía, 63(2), 117-140.

Gallardo, K. E., Alvarado, M. A., Lozano, A., López, C. S. y Gudiño, S. (2017). Materiales digitales para fortalecer el aprendizaje disciplinar en educación media superior: Un estudio para comprender cómo se suscita el cambio educativo. REICE. Revista Iberoamericana sobre Calidad, Eficacia y Cambio en Educación, 15(2), 89-109. https://doi.org/10.15366/reice2017.15.2.005

Gil, D. y Vilches, A. (2017). Educación para la sostenibilidad y educación en derechos humanos. Teoría de la Educación, 29(1), 79-100. https://doi.org/10.14201/teoredu29179100

González, N. y Rossi, A. (2015). Conocimiento pedagógico del contenido para la enseñanza del tema mitosis: Un estudio de casos con docentes universitarios de Argentina. Didáctica de las Ciencias Experimentales y Sociales, 29, 215-232. https://doi.org/10.7203/dces.29.4991

Gore, J. M. y Gitlin, A. D. (2007). Visioning the academic-teacher divide: Power and knowledge in the educational community. Teachers and Teaching, 10(1), 35-58. https://doi.org/10.1080/13540600320000170918 
Greca, I. M. y El-Hani, C. N. (2015). Docentes e investigadores en comunidades virtuales de práctica para el desarrollo profesional docente y la mejoría de la enseñanza de las ciencias. Revista de Enseñanza de la Física, 27(1), 7-18.

Gutiérrez, A., Muñoz de Corrales, E. y del Campo, R. (2017). La reflexión de los docentes en la enseñanza de las ciencias en primaria. Investigación en la Escuela, 91, 1-18. https://doi.org/10.12795/IE.2017.i91.01

Harlen, W. (2015). Trabajando con las grandes ideas de la educación en ciencias. Roma: Red Global de Academias de Ciencias.

Íñiguez-Porras, F. J. y Puigcerver-Oliván, M. (2013). Una propuesta didáctica para la enseñanza de la genética en la educación secundaria. Revista Eureka sobre Enseñanza y Divulgación de las Ciencias, 10(3), 307-327.

https://doi.org/10.25267/Rev_Eureka_ensen_divulg_cienc.2013.v10.i3.02

Ion, G. y Iucu, R. (2014). Professionals' perceptions about the use of research in educational practice. European Journal of Higher Education, 4(4), 334-347.

https://doi.org/10.1080/21568235.2014.899154

Jiménez-Aleixandre, P. (2008). La publicación como proceso de diálogo y aprendizaje: El papel de artículos y revistas en didáctica de las ciencias. Enseñanza de las Ciencias 26(3), 311-320.

Jong, O. (2007). Teaching practice and research in chemistry education: Living apart or together? En M. Izquierdo, A. Caamaño y M. Quintanilla (Eds.), Investigar en la enseñanza de la química. Nuevos horizontes: contextualizar y modelizar (pp.164-171). Barcelona: Universidad Autónoma de Barcelona.

Kaestle, C. (1993). The awful reputation of education research. Educational Researcher, 22(1), $23-31$. https://doi.org/10.2307/1177303

Lara, M., Förster, C. y Gorichon, S. (2018). Transferencia de la investigación educacional a la formación inicial de profesores. Calidad en la Educación, 27, 178-204.

https://doi.org/10.31619/caledu.n27.223

Lavis, J. N., Robertson, D., Woodside, J. M., McLeod, C. B. y Abelson, J. (2003). How can research organizations more effectively transfer research knowledge to decision makers? Milbank Quarterly, 81(2), 221-248. https://doi.org/10.1111/1468-0009.to1-1-00052

Lewis, J., Leach J. y Wood-Robinson C. (2000). Chromosomes: The missing link-people's understanding of mitosis, meiosis, and fertilisation. Journal of Biological Education, 34, 189199. https://doi.org/10.1080/002 19266.2000.9655717

Lupión Cobos, T. y Martín Gámez, C. (2016). Desarrollo profesional docente de profesorado de secundaria en una experiencia de innovación mediante investigaciones escolares. Revista Eureka sobre Enseñanza y Divulgación de las Ciencias, 13(3), 686-704. http://dx.doi.org/10.25267/Rev_Eureka_ensen_divulg_cienc.2016.v13.i3.13

Magnusson, S., Krajcik, J. y Borko, H. (1999). Nature, sources, and development of pedagogical content knowledge for science teaching. En J. Gess-Newsome y N. G. Lederman (Eds.), Examining pedagogical content knowledge (pp. 95-132). Dordrecht: Kluwer Academic Publishers. https://doi.org/10.1007/0-306-47217-1_4

Manzano-García, B. (2016). La investigación en educación llevada a la práctica para la mejora en los procesos educativos. En M. Tomé Fernández y B. Manzano-García (Coords.), Investigación en la práctica docente (pp. 3-18). Madrid: Ediciones Nobel.

Martínez, R. A. (2007). La investigación en la práctica educativa: Guía metodológica de investigación para el diagnóstico y evaluación en los centros docentes. Madrid: Secretaría General Técnica, Centro de Publicaciones, Ministerio de Educación y Ciencia. 
Morales, P. (2010). Investigación e innovación educativa. REICE. Revista Iberoamericana sobre Calidad, Eficacia y Cambio en Educación, 8(2), 47-73.

Muñoz-Repiso, M. (2004). Investigación, política y prácticas educativas. En J. Torre Puente y E. Gil Coria (Eds.), Hacia una enseñanza universitaria centrada en el aprendizaje (pp. 405-429). Madrid: Universidad Pontificia Comillas.

Muñoz Martínez, M. y Garay, F. (2015). La investigación como forma de desarrollo profesional docente: Retos y perspectivas. Estudios Pedagógicos, 41(2), 389-399. https://doi.org/10.4067/S07 18-07052015000200023

Murillo, F. J. y Hernández-Castilla, R. (2011). Hacia un concepto de justicia social. REICE. Revista Iberoamericana sobre Calidad, Eficacia y Cambio en Educación, 9(4), 7-23.

Murillo, F. J. y Krichesky, G. J. (2012). El proceso de cambio escolar: Una guía para impulsar y sostener la mejora de las escuelas. REICE. Revista Iberoamericana sobre Calidad, Eficacia y Cambio en Educación, 1O(1), 27-43.

Murillo, F. J. y Perines, H. (2017). Cómo los docentes no universitarios perciben la investigación educativa. Revista Complutense de Educación, 28(1), 81-99. https://doi.org/10.5209/rev_RCED.2017.v28.n1.48800

Murillo, F. J., Martínez-Garrido, C. y Belavi, G. (2017). Sugerencias para escribir un buen artículo científico en educación. REICE. Revista Iberoamericana sobre Calidad, Eficacia y Cambio en Educación, 15(3), 5-34. https://doi.org/10.15366/reice2017.15.3.001

OCDE. (2009). Working out change. Systemic innovation in vocational education and training. París: OCDE Publishing. https://doi.org/10.1787/9789264075924-en

Oliva, J. M. (2005). Sobre el estado actual de la revista enseñanza de las ciencias y algunas propuestas de futuro. Enseñanza de las Ciencias, 23(1), 123-132.

Oliva, J. M. (2011). Dificultades para la implicación del profesorado de educación secundaria en la lectura, innovación e investigación en didáctica de las ciencias (I): El problema de la inmersión. Revista Eureka sobre Enseñanza y Divulgación de las Ciencias, 8(1), 41-53. https://doi.org/10.25267/Rev_Eureka_ensen_divulg_cienc.2011.v8.i1.04

Oliva, J. M. (2012). Dificultades para la implicación del profesorado de educación secundaria en la lectura, innovación e investigación en didáctica de las ciencias (II): El problema del manos a la obra. Revista Eureka sobre Enseñanza y Divulgación de las Ciencias, 9(2), 241-251. https://doi.org/10.25267/Rev_Eureka_ensen_divulg_cienc.2012.v9.i2.06

Oliva, J. M. (2017). Sobre el difícil equilibrio de compaginar criterios de difusión y de transferencia en una revista sobre educación científica. Revista Eureka sobre Enseñanza y Divulgación de las Ciencias, 14(1), 1-2. https://doi.org/10.25267/Rev_Eureka_ensen_divulg_cienc.2017.v14.i1.01

Pedró, F. (2015). Las políticas de investigación e innovación en educación: Una perspectiva supranacional. Bordón. Revista de Pedagogía, 67(1), 39-56. https://doi.org/10.13042/Bordon.2015.67103

Pérez Martín, J.M. y Aquilino, M. (2017). Conocimiento del ciclo celular en docentes de ciencias. En M. González Montero de Espinosa, A. Baratas Díaz y A. Brandi Fernández (Eds.), Actas de las IV Jornadas sobre investigación y didáctica en ESO y Bachillerato (pp. 403-412). Madrid: Santillana.

Pérez Martín, J. M. y Bravo Torija, B. (2018). Experiencias para una alfabetización científica que promueva la justicia ambiental en distintos niveles educativos. Revista Internacional de Educación para la Justicia Social, 7(1), 119-140. https://doi.org/10.15366/riejs2018.7.1.006 
Pérez Martín, J. M. y Martínez Luna, S. (2017). Ideas alternativas sobre el trabajo fin de grado en estudiantes de Magisterio. ReiDoCrea, 6, 246-259.

Perines, H. (2018). ¿Por qué la investigación educativa no impacta en la práctica docente? Estudios sobre Educación, 34, 9-27. https://doi.org/10.15581/004.34.9-27

Porlán, R. (2018). Didáctica de las ciencias con conciencia. Enseñanza de las ciencias: Revista de Investigación y Experiencias Didácticas, 36(3), 5-22. https://doi.org/10.5565/rev/ensciencias.2795

Robles, A., Solbes, J., Cantó, J. R. y Lozano, O. R. (2015). Actitudes de los estudiantes hacia la ciencia escolar en el primer ciclo de la enseñanza secundaria obligatoria. Revista Electrónica de Enseñanza de las Ciencias, 14(3), 361-376. https://doi.org/10.5565/rev/ensciencias.2795

Ruíz-González, C., Banet, E. y López Banet, L. (2017). Conocimientos de los estudiantes de secundaria sobre herencia biológica: Implicaciones para su enseñanza. Revista Eureka sobre Enseñanza y Divulgación de las Ciencias, 14(3), 550-569. http://dx.doi.org/10.25267/Rev_Eureka_ensen_divulg_cienc.2017.v14.i3.04

Ruiz García, A. y Valero Aguayo, L. (2018). Efectos del JCR en la calidad de las revistas españolas de psicología: Un selfi en 2017. Apuntes de Psicología, 36(2), 63-74.

Russell, T. y Martin, A. K. (2014). Learning to teach science. En N. G. Lederman y S. K. Abell (Eds.), Handbook of research on science education (pp. 885-902). Abingdon: Routledge.

Sancho, J. M. (2010). Del sentido de la investigación educativa y la dificultad de que se considere para guiar las políticas y las prácticas. REICE. Revista Iberoamericana sobre Calidad, Eficacia y Cambio en Educación, 8(2), 34-46.

Sanmartí, N. (2008). Contribuciones y desafíos de las publicaciones del área de educación en ciencias en la construcción y consolidación de la identidad del área: La experiencia de la revista enseñanza de las ciencias. Enseñanza de las Ciencias, 26(3), 301-310.

Solbes, J., Domínguez-Sales, M. C., Fernández-Sánchez, J., Furió, C., Cantó, J. y Guisasola, J. (2013). ¿El profesorado de física y química incorpora los resultados de la investigación en didáctica? Didáctica de las Ciencias Experimentales y Sociales, 27, 155-178.

https://doi.org/10.7203/dces.27.2617

Solbes, J., Fernández-Sánchez, J., Domínguez-Sales, M. C., Cantó, J. y Guisasola, J. (2018). Influencia de la formación y la investigación didáctica del profesorado de ciencias sobre su práctica docente. Enseñanza de las Ciencias, 36(1), 25-44.

Solbes, J. y Gavidia, V. (2013). Análisis de las especialidades de física y química y de biología y geología del máster de profesorado de educación secundaria de la universidad de valencia. Revista Eureka sobre Enseñanza y Divulgación de las Ciencias, 10, 582-593. https://doi.org/10.25267/Rev_Eureka_ensen_divulg_cienc.2013.v10.iextra.07

Solís, E. (2011). ¿Cómo integrar la investigación, la innovación y la práctica en la enseñanza de las ciencias? Alambique. Didáctica de las Ciencias Experimentales, 68, 80-88.

Strieder, R. B., Bravo-Torija, B. y Gil-Quílez, M. J. (2017). Ciencia-tecnología-sociedad: ¿Qué estamos haciendo en el ámbito de la investigación en educación en ciencias? Enseñanza de las Ciencias, 35(3), 29-49. https://doi.org/10.5565/rev/ensciencias.2232

Talanquer, V. (2013). Progresiones de aprendizaje: Promesa y potencial. Educación Química, 24(4), 362-364. https://doi.org/10.1016/So 187-893X(13)72488-1

van Schaik, P., Volman, M., Admiraal, W. y Schenke, W. (2018). Barriers and conditions for teachers' utilisation of academic knowledge. International Journal of Educational Research, 90, 50-63. https://doi.org/10.1016/j.ijer.2018.05.003 
Vanderlinde, R. y Van Braak, J. (2010). The gap between educational research and practice: Views of teachers, school leaders, intermediaries and researchers. British Educational Research Journal, 36(2), 299-316. https://doi.org/10.1080/01411920902919257

Wandersman, A., Duffy, J., Flaspohler, P., Noonan, R., Lubell, K., Stillman, L. y Saul, J. (2008). Bridging the gap between prevention research and practice: The interactive systems framework for dissemination and implementation. American Journal of Community Psychology, 41(4), 171-181. https://doi.org/10.1007/s 10464-008-9174-Z

Williams, D. y Coles, L. (2007). Teachers' approaches to finding and using research evidence: An information literacy perspective. Educational Research, 49(2), 185-206. https://doi.org/10.1080/00131880701369719

Wissiak, K. S. y Ferk, V. (2013). Bridging the gap between educational research and school practice through cooperation of university and primary school teachers. Procedia-Social and Behavioral Sciences, 106, 576-584. https://doi.org/10.1016/j.sbspro.2013.12.066

\section{Breve CV de los autores}

\section{Tamara Esquivel-Martín}

Doctoranda en Educación en la Universidad Autónoma de Madrid (UAM). Investigadora en la línea de Didáctica de las Ciencias Experimentales y las Matemáticas para el Cambio Educativo y la Justicia Social. Con Máster en Formación de Profesorado de Educación Secundaria Obligatoria y Bachillerato (UAM). Graduada en Biología Sanitaria por la Universidad de Alcalá. Ha sido profesora de los grados de Educación Infantil y Primaria en la Universidad Isabel I y la Universidad Católica San Antonio de Murcia. Ha participado en proyectos de investigación e innovación nacionales e internacionales que han derivado en diversas publicaciones y comunicaciones científicas sobre la enseñanzaaprendizaje de Biología. ORCID ID: https://orcid.org/0000-0002-0739-9099. Email: tamara.esquivel@estudiante.uam.es

\section{Beatriz Bravo-Torija}

Doctora en Didáctica de las Ciencias Experimentales y Licenciada en Biología por la Universidad de Santiago de Compostela (USC). Profesora del Departamento de Didácticas Específicas (área de Didáctica de las Ciencias Experimentales) de la UAM. Anteriormente ha sido profesora en las facultades de Educación de las universidades de Santiago de Compostela, Granada y Zaragoza, impartiendo docencia de Máster y en los grados de Educación Infantil y Primaria. Ha participado en proyectos nacionales e internacionales de los que derivan diversas comunicaciones a congreso y publicaciones científicas indexadas en bases de datos de alto impacto sobre la argumentación en problemas sociocientíficos; y es revisora en Enseñanza de las Ciencias, Science Education y Journal of Biological Education. ORCID ID: https://orcid.org/O000-0001-6236-6807. Email: beatriz.bravo@uam.es

\section{José Manuel Pérez Martín}

Doctor en Biología y Licenciado en Biología por la UAM. Profesor del Departamento de Didácticas Específicas (área de Didáctica de las Ciencias Experimentales) de la UAM. 
Anteriormente ha sido profesor en las facultades de Ciencias de la UAM y de Educación de la Universidad de Camilo José Cela y de la Universidad Internacional de La Rioja (UNIR). Ha impartido docencia en varios grados y másteres de Ciencias y de Educación. Ha participado en proyectos nacionales e internacionales de los que derivan diversas comunicaciones a congreso y publicaciones científicas sobre los efectos tóxicos de los contaminantes ambientales en la salud humana y ambiental, y sobre la enseñanza de las ciencias en revistas nacionales e internacionales indexadas en bases de datos de alto impacto. ORCID ID: https://orcid.org/0000-0002-0658-9050. Email: josemanuel.perez@uam.es 